\title{
Kernos
}

Revue internationale et pluridisciplinaire de religion grecque antique

$33 \mid 2020$

Varia

\section{Kinship in Ancient Athens. An Anthropological Analysis}

Jérôme Wilgaux

\section{(2) OpenEdition \\ Journals}

Édition électronique

URL : https://journals.openedition.org/kernos/3571

DOI : 10.4000/kernos.3571

ISSN : 2034-7871

Éditeur

Centre international d'étude de la religion grecque antique

Édition imprimée

Date de publication : 31 décembre 2020

Pagination : 334-336

ISBN : 978-2-87562-264-8

ISSN : 0776-3824

Référence électronique

Jérôme Wilgaux, «Kinship in Ancient Athens. An Anthropological Analysis », Kernos [En ligne], 33 |

2020, mis en ligne le 31 décembre 2020, consulté le 06 décembre 2022. URL : http://

journals.openedition.org/kernos/3571; DOI : https://doi.org/10.4000/kernos.3571

Ce document a été généré automatiquement le 6 décembre 2022

Tous droits réservés 


\title{
Kinship in Ancient Athens. An Anthropological Analysis
}

\author{
Jérôme Wilgaux
}

\section{RÉFÉRENCE}

Sally C. HUMPHREYS, Kinship in Ancient Athens. An Anthropological Analysis, Oxford, Oxford University Press, 2018. 2 vol. 19,2 × 25,3 cm, 1457 p. ISBN : 978-0-19-878825-6 ; 978-0-19878826-3.Doralice FABIANO, Senza paradiso. Miti e credenze sull'aldilà greco, Bologna, Il Mulino, 2019. 1 vol. 13,5 × 21,2 cm, 278 p. (Antropologia del mondo antico, 12). ISBN : 978-88-15-27996-5.

Sarah C. (Sally) Humphreys (S.H.), aujourd'hui professeur émérite d'histoire à l'Université du Michigan (Ann Arbor), a tout au long de sa carrière académique consacré ses recherches aux institutions politiques, sociales et religieuses des cités grecques antiques, plus particulièrement aux époques archaïque et classique; une partie de ses travaux a d'ores et déjà été réunie dans de précédentes publications (notamment Anthropology and the Greeks, 1978; The Family, Women and Death, 1983; The Strangeness of Gods: Historical Perspectives on the Interpretation of Athenian Religion, 2004), mais l'ouvrage publié en 2018 par les presses universitaires d'Oxford constitue assurément un ouvrage hors-norme, une somme monumentale, synthèse de plusieurs décennies de travail centrées sur l'étude de la parenté athénienne. Pour en donner une première présentation rapide, l'ouvrage est composé de deux volumes. Le premier (xxi, p. 1-533) est divisé en chapitres portant sur les aspects juridiques et les interactions socio-économiques (adoption, tutelle, mariage entre proches, propriété, solidarité et conflits familiaux), les rituels (rites de passage et commémoratifs) et l'imbrication de la parenté dans la vie politique (la dimension politique des mariages par ex.). Le second volume (vi, p. 534-1457) est pour sa part consacré aux corporate groups (c'est-à-dire aux groupes appréhendés en tant que personnes morales), de nature tout à la fois parentale et politique (tribus, trittyes, phratries, genē) ainsi qu'aux dèmes (auxquels sont consacrés près de 650 pages). Dans ce second volume, cinq appendices reviennent sur 
des documents controversés. Une bibliographie imposante (p. 1231-1367) ainsi que des indices très détaillés (thèmes, noms, sources, p. 1369-1457) complètent cet ensemble.

2 L'ambition de l'étude est exposée dans les premières pages: se focalisant sur la cité grecque la mieux documentée, l'Athènes des $\mathrm{VI}^{\mathrm{e}}-\mathrm{IV}^{\mathrm{e}} \mathrm{s}$., et s'appuyant notamment les apports récents de l'épigraphie et des recherches prosopographiques (les apports du travail réalisé dans le cadre du Lexicon of Greek Personal Names sont ici manifestes), la recherche proposée souhaite rendre compte des normes et pratiques (patterns of behaviour) d'une société antique dans le domaine de la parenté, et ce dans une démarche tout à la fois historique (ainsi attentive à rendre compte des évolutions) et anthropologique (en ce sens, mobilisant les outils méthodologiques et conceptuels forgés par l'anthropologie de la parenté depuis le XIXe siècle).

3 La progression générale de l'ouvrage témoigne clairement de la manière dont cette ambition est mise en œuvre. Commençant par l'analyse des législations archaïques (les lois attribuées à Dracon et Solon), l'étude replace ainsi les pratiques familiales dans leur cadre primordial, normatif et civique : c'est bien la communauté politique dans son ensemble qui se donne un droit de regard sur les comportements parentaux de ses membres - et en particulier de ses élites - afin notamment de contrôler la continuité des oikoi, leur composition et leurs modes de transmission. Cette imbrication du parental et du politique constitue l'un des fils rouges de cette étude, ainsi que le démontre également la composition du second volume, consacré en totalité aux subdivisions civiques qui font ainsi l'objet d'une présentation détaillée accordant toute son importance à la documentation prosopographique et aux relations non seulement parentales mais aussi locales (relations de voisinage), territoriales et communautaires ; de fait, l'importance des dèmes dans les vies familiales constitue l'un des thèmes majeurs de l'ouvrage.

Une fois ce cadre civique et normatif présenté, le premier volume aborde successivement, ainsi qu'il est attendu de toute étude anthropologique, les domaines de la filiation, du mariage et de la transmission des patrimoines : les premiers chapitres en présentent les principes, mais chaque thème est étudié en entrant dans le détail des conflits familiaux documentés, de sorte que si les parcours individuels et familiaux s'inscrivent dans un cadre communautaire commun, ils permettent dans le même temps d'en mettre en évidence les tensions et les contradictions (entre liens patrilinéaires et bilatéraux notamment), ainsi que la plus ou moins grande liberté de manœuvre accordée aux stratégies individuelles ou familiales. La longueur du chapitre relatif aux conflits familiaux (chap. VII, «Disputes», p. 185-269) témoigne de leur importance dans la réflexion proposée : la question de la manière dont se nouent ou se dénouent les solidarités familiales constitue ainsi clairement le second fil rouge de cet ouvrage.

5 Sans surprise de la part d'une chercheuse ayant consacré de nombreuses études à la religion grecque, la dimension religieuse de la construction des identités et des appartenances constitue le troisième fil rouge de l'ouvrage et fait plus particulièrement l'objet de la deuxième partie de l'ouvrage («Ritual», p. 261-422); sont ainsi plus particulièrement étudiés les rituels d'intégration (ou d'exclusion, cf. par ex. l'apokēryxis, mentionnée à plusieurs reprises dans l'ouvrage) dans les communautés familiales et civiques (les phratries notamment), de la dation des noms jusqu'au mariage, ainsi que les funérailles et autres rituels de commémoration; l'imbrication du parental et du religieux est également étudiée dans le cadre des fêtes et associations religieuses. Dans 
d'autres parties de l'ouvrage, sont également étudiées les tablettes de malédiction, replacées dans leur contexte politique, ou bien encore la dimension religieuse des subdivisions civiques : de fait, tout au long du second volume, la présentation détaillée des genē, phratries, tribus et dèmes en souligne les activités religieuses et revient fréquemment sur des dossiers documentaires centrés sur des fêtes et rituels (lois sacrées...).

6 Ainsi que nous l'avons vu, les analyses proposées ne se limitent pas aux seuls cadres parentaux, insistant par ex. sur l'importance des liens politiques et civiques dans les parcours de vie individuels; il en est de même en ce qui concerne les pratiques religieuses, et s'arrêtant par exemple un instant, p. 422, sur de possibles prolongements de ces observations, S.H. relève combien la documentation athénienne, du $\mathrm{VI}^{\mathrm{e}}$ au $\mathrm{IV}^{\mathrm{e}} \mathrm{s}$. puis à l'époque hellénistique, se complexifie et rend de plus en plus confus, à nos yeux, la distinction entre privé et public ou bien encore les clivages statutaires; les liens de parenté s'entremêlent ainsi avec d'autres modes d'association, plus ou moins identifiables selon nos sources. À plusieurs reprises, S.H. met d'ailleurs en évidence la manière dont les approches historiennes ont évolué sur ces points au cours des dernières décennies, au fil des découvertes archéologiques et épigraphiques notamment, et se distancie alors de certains de ses propres travaux, qu'elle considère aujourd'hui comme ayant pu insuffisamment prendre la mesure des problèmes méthodologiques posés par l'étude des croyances et des pratiques religieuses, dans le cadre des funérailles, des fêtes ou autres rituels; ces multiples remarques, dans le cadre d'une démarche réflexive, sur la manière dont les recherches sur les cités grecques ont pu être menées et évoluer depuis les années 1970, l'explicitation de leurs enjeux et de leurs limites, sont bien sûr d'un intérêt majeur pour le lecteur.

7 Les analyses proposées tout au long de ces chapitres témoignent donc, s'il en était encore besoin, combien les faits de parenté sont au cœur des constructions civiques grecques, et l'ouvrage rassemble en ce sens une masse impressionnante de matériaux et de commentaires, touchant à de multiples aspects de la vie quotidienne athénienne. Tous les documents majeurs relatifs à la parenté athénienne - les plaidoyers notamment - font l'objet au fil des pages de commentaires approfondis, les reconstitutions prosopographiques replacées dans le cadre des dèmes et tribus constituent des apports indéniables, et plusieurs dizaines de stemmata et autres graphiques de parenté complètent ces études. À ce titre, la synthèse publiée par S.H. constitue donc un outil de travail désormais indispensable pour toutes recherches consacrées à la société grecque ancienne et à la cité d'Athènes.

8 L'ouvrage n'est pas cependant sans susciter de nombreuses interrogations. Du fait même de ses dimensions et de la multiplicité des thèmes abordés, chaque partie ou sous-partie se développe de manière plus ou moins indépendante, et le lecteur peine à dégager des logiques d'ensemble. De ce point de vue, il est dommage que S.H. n'ait pas proposé en conclusion de l'ouvrage son propre bilan méthodologique et conceptuel (voir cependant les remarques développées aux pages 252-260).

Qui plus est, le domaine de la parenté s'est profondément transformé dans ses thématiques et ses problématiques au cours des dernières décennies, et de ce point de vue l'ouvrage parait quelque peu en décalage par rapport aux débats les plus actuels. S.H. en a assurément conscience et nous précise d'ailleurs en introduction que deux des pré-lecteurs sollicités par la maison d'édition avaient jugé la structure de l'ouvrage quelque peu «outdated», sans que cela ne l'incite pour autant à modifier son projet 
(cf. p. 3, «I am not clear that these changes in anthropological focus seriously affect the utility of my analysis »).

10 Par-delà l'analyse d'une structure sociale, les études de la parenté se donnent aujourd'hui des ambitions contrastées, avec notamment la volonté de rendre compte de la manière dont une société se reproduit en choisissant, en « fabriquant » ses membres, en les dotant de biens et de " qualités » particulières, et ce de manière hiérarchisée ; les faits de parenté participent ainsi du processus de construction, de reproduction et de légitimation des distinctions sociales et des rapports de pouvoir, le plus souvent en les naturalisant. Dans ces perspectives, le domaine de la parenté fait l'objet d'approches non plus seulement sociales mais aussi culturelles, et les rapports de parenté deviennent indissociables des multiples clivages qui structurent une société, pour lesquels les gender ou bien encore les subaltern studies proposent aujourd'hui de nouvelles approches. Concrètement, en vérité, les analyses proposées par S.H. témoignent (bien plus que ses propos introductifs ne semblent le suggérer) de ces évolutions, abordant ici et là les questions liées aux représentations de la procréation et de la parenté, à la sexualité, aux constructions de la personne et des identités collectives, à la dimension parentale du rapport servile, etc. Mais c'est bien la grille de lecture globale qui reste incertaine: entre pratiques et discours, structures et stratégies, communauté et singularité..., les faits de parenté relèveraient-ils d'une définition et d'une synthèse toujours inachevées?

\section{AUTEURS}

JÉRÔME WILGAUX

Université de Nantes 